\title{
MyD88-dependent IL-1 receptor signaling is essential for gouty inflammation stimulated by monosodium urate crystals
}

Chun-Jen Chen, ${ }^{1}$ Yan Shi, ${ }^{1}$ Arron Hearn, ${ }^{1}$ Kate Fitzgerald,, ${ }^{2}$ Douglas Golenbock, ${ }^{2}$ George Reed, ${ }^{2}$ Shizuo Akira, ${ }^{3}$ and Kenneth L. Rock ${ }^{1}$

\begin{abstract}
1Department of Pathology and 2Department of Medicine, University of Massachusetts Medical School, Worcester, Massachusetts, USA.
\end{abstract} ${ }^{3}$ Department of Host Defense, Research Institute for Microbial Diseases, Osaka University, Osaka, Japan.

\begin{abstract}
While it is known that monosodium urate (MSU) crystals cause the disease gout, the mechanism by which these crystals stimulate this inflammatory condition has not been clear. Here we find that the Toll/IL-1R (TIR) signal transduction adaptor myeloid differentiation primary response protein 88 (MyD88) is required for acute gouty inflammation. In contrast, other TIR adaptor molecules, TIRAP/Mal, TRIF, and TRAM, are not required for this process. The MyD88-dependent TLR1, $-2,-4,-6,-7,-9$, and -11 and IL-18 receptor (IL-18R) are not essential for MSU-induced inflammation. Moreover, MSU does not stimulate HEK cells expressing TLR1-11 to activate NF- $\kappa B$. In contrast, mice deficient in the MyD88-dependent IL-1R showed reduced inflammatory responses, similar to those observed in MyD88-deficient mice. Similarly, mice treated with IL-1 neutralizing antibodies also showed reduced MSU-induced inflammation, demonstrating that IL-1 production and IL-1R activation play essential roles in MSU-triggered inflammation. IL-1R deficiency in bone marrow-derived cells did not affect the inflammatory response; however, it was required in nonbone marrow-derived cells. These results indicate that IL-1 is essential for the MSU-induced inflammatory response and that the requirement of MyD88 in this process is primarily through its function as an adaptor molecule in the IL-1R signaling pathway.
\end{abstract}

\section{Introduction}

Gout is one of the oldest recorded afflictions of humans. It occurs in individuals with hyperuricemia when monosodium urate (MSU) crystals precipitate in tissues and stimulate acute inflammation (1). Although uric acid was identified in gout in the late 1700s (2) and shown to be the causative agent of this disease in 1899 (3), we have not known exactly how MSU crystals trigger acute inflammation. Several different mechanisms have been suggested as underlying this inflammatory response. It has been proposed that MSU crystals induce inflammation by mechanically disrupting phagolysosomes and killing neutrophils, with the attendant release of destructive enzymes (4). Alternatively, it has been suggested that extracellular MSU crystals activate complement (5-9) and other extracellular enzymes such as bradykinin and kallikrein and thereby generate proinflammatory mediators (10). Yet another potential mechanism is that phagocytosis of MSU crystals may stimulate the production of eicosanoids and reactive oxygen species (11-13) as well as proinflammatory cytokines (e.g., TNF- $\alpha$ [ref. 14], IL-1 $\beta$ [ref. 15-17], and IL-6 [ref. 18]). In addition, increased levels of chemokine monocyte chemoattractant protein-1 (MCP-1) (19) and the chemotactic factors for neutrophils, such as the C-X-C chemokines IL-8 (in humans) (20), keratinocyte-derived chemokine (KC) and macrophage inflammatory protein-2 (MIP-2) (in rodents) $(17,21)$, and

Nonstandard abbreviations used: HCM, hybridoma culture medium; IL-1R, IL-1 receptor; KC, keratinocyte-derived chemokine; Mal, MyD88 adaptor-like protein; MIP-2, macrophage inflammatory protein-2; MSU, monosodium urate; MyD88, myeloid differentiation primary response protein 88 ; PEC, peritoneal exudate cell; TIR, Toll/IL-1R; TIRAP, TIR domain-containing adaptor protein; TRAM, TRIF-related adaptor molecule; TRIF, TIR domain-containing adaptor-inducing IFN- $\beta$. Conflict of interest: The authors have declared that no conflict of interest exists. Citation for this article: J. Clin. Invest. 116:2262-2271 (2006). doi:10.1172/JCI28075. myeloid-related proteins S100A8/A9 (22) and S100A12 (23), have also been detected in synovial fluid of patients with acute gout or in murine gout models. The exact contributions of these different mechanisms to gouty inflammation and the mechanisms by which cellular responses were triggered have not been fully understood.

Recently, it was also found that urate release from injured cells provides an adjuvant signal that alerts the immune system to danger (24). Adjuvants were originally discovered in the early 1920 s as immunostimulatory molecules $(25,26)$ and later shown to be essential components for successful immunization. Until recently, most known adjuvants were molecules of microbial origin whose chemically distinct structures were recognized by receptors on sentinel cells of the immune system, such as macrophages and dendritic cells $(27,28)$. As a consequence of this recognition, the immune cells are stimulated to begin to combat the microbe directly and also to recruit further immune responses. It is believed that this recognition system evolved to allow the immune system to identify and selectively respond to infections (27). Urate represented a new class of adjuvant molecules being produced by the host itself and of a distinct chemical structure. This helped to explain how the immune system can respond to stimuli such as tumors and transplants that lack microbial components (29). It was postulated that this role of MSU in triggering the innate immune system might underlie the pathogenesis of gout: crystals deposit in tissues of hyperuricemic patients and signal "danger" (24).

While we don't fully understand how MSU stimulates its biological effects, the mechanisms underlying activity of microbial adjuvants are much better understood. These molecules are recognized by pattern recognition receptors such as TLRs (28). Once stimulated, the TLRs associate with intracellular adaptor molecules (Toll/IL-1R [TIR] adaptors) to initiate a signaling cascade that 
A

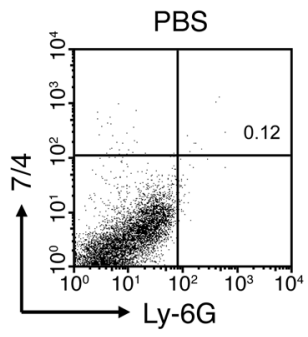

B

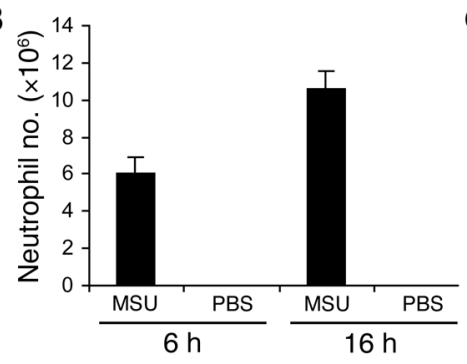

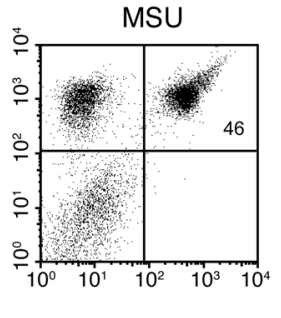

C

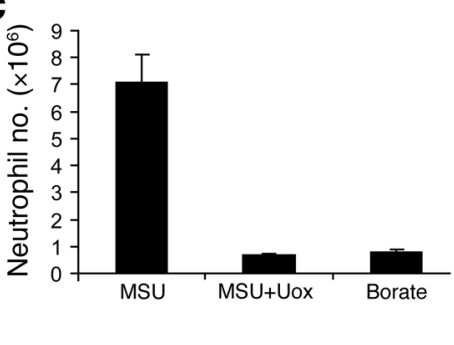

\section{Figure 1}

MSU induces peritonitis in mice. (A and B) C57BL/6 mice were challenged i.p. with $3 \mathrm{mg}$ of MSU crystals. Mice challenged with PBS served as negative controls. Peritoneal cells were stained for $L y-6 G$ and $7 / 4$ expression and analyzed by flow cytometry. (A) Representative dot plots of Ly-6G and $7 / 4$ expression on peritoneal cells in animals injected 6 hours earlier with PBS or MSU. The Ly- $6 \mathrm{G}^{+} 7 / 4^{+}$ gate represents neutrophils. (B) At indicated time points, neutrophil numbers in PECs were determined by multiplying the total cell numbers by the percentage of $L y-6 G+7 / 4^{+}$ cells $(n=3)$. (C) C57BL/6 mice were challenged i.p. with $1.5 \mathrm{mg}$ of MSU crystals, $1.5 \mathrm{mg}$ of uricase-digested MSU (MSU+Uox), or borate buffer. At 16 hours after challenge, neutrophil numbers in the PECs were determined $(n=3)$. Data shown are representative of 3 or more experiments. activates proinflammatory transcription factors such as NF- $\mathrm{KB}$ and IFN regulatory factors (IRFs) (30). Given that microbial molecules and MSU both have adjuvant and proinflammatory properties, we hypothesized that these 2 classes of immunologically active molecules might signal through similar pathways. This study was initiated to test this hypothesis.

\section{Results}

The role of TLRs in MSU-stimulated acute inflammation. The hallmark of the response to MSU in gout is acute inflammation with an infiltration of neutrophils at the site of crystal deposition in vivo. Although in gout MSU crystals most typically form in joints, causing arthritis, deposition elsewhere either pathologically or by experimental injection induces acute inflammation (31-33). To study the acute inflammatory response to MSU crystals, we injected them into the peritoneal cavity in mice. Six hours after injection, we analyzed the exudate cells in the peritoneum. There was a marked influx of cells that express the neutrophil markers Ly-6G and 7/4 into this site (Figure 1A), and the number of neutrophils further increased at 16 hours after MSU injection (Figure 1B). Using this model, we investigated how MSU crystals stimulated acute inflammation.

One of the principal sets of receptors for microbial molecules that stimulate inflammation and adjuvant effects are the TLRs. To test the hypothesis that MSU signals through a mechanism similar to that of microbial proinflammatory molecules, we first examined whether MSU stimulated inflammation in mice deficient in various TLRs. MSU crystals were injected into the peritoneum of all of the available TLR-deficient mice, and the acute inflammatory response was evaluated by quantifying the influx of neutrophils. The MSU-induced inflammation was not reduced in TLR1, $-2,-3$, $-4,-6,-7,-9$, and $-11-$ deficient mice (Figure 2). Statistical analysis of the results of 3 independent experiments using 6-9 total mice (except for $n=3$ for $T L R 7^{-1-}$ mice) showed no significant reduction in neutrophil infiltration in TLR-deficient animals compared with the WT animals; in fact, we actually observed significant increases in neutrophil influx in mice deficient of TLR1, -3, -4, -6, and -9. Therefore, none of these TLRs are required for responses to MSU and, if TLRs play any role, it is so minor as to be undetectable with the numbers of mice that we analyzed. Our results are different from what was reported recently by Liu-Bryan et al. (34): that MSU-stimulated neutrophil influx in a subcutaneous air pouch model is reduced in TLR2 $2^{-/-}$and TLR4 $4^{-/}$mice. To further examine whether TLR2 and TLR4 are involved in the inflammatory response stimulated by MSU, we injected MSU i.p. into TLR2/4 double-deficient mice and found that neutrophil infiltration into the peritoneum was not affected compared with that in WT mice (Figure 2I), demonstrating that TLR2 and TLR4 are not essential for MSU-induced inflammation. The TLR10 gene in mice is not functional due to a retroviral insertion and therefore cannot be participating in the MSU response (35). Mice deficient for TLR5 and TLR 8 are not yet available, so these experiments by themselves do not rule out a role for these 2 TLRs.

To confirm the above results and also rule out the possibility that MSU crystals stimulate TLR5, TLR8, or more than one TLR, we performed a second set of gain-of-function experiments. Stimulation of TLRs leads to the activation of the transcription factor NF-кB through the myeloid differentiation primary response protein 88-dependent (MyD88-dependent) pathway. Accordingly, activation of a NF- $\kappa \mathrm{B}$ luciferase construct in TLR-expressing HEK cells has been used as a sensitive assay for TLR function (36). Therefore, we used this experimental system to determine whether MSU crystals would stimulate HEK cells expressing various endogenous or transfected TLRs to activate a NF-אB-luciferase reporter gene. HEK cells naturally express TLR1, TLR5, and TLR6 (37). When these cells were transfected with TLR2, $-3,-7,-8$, or -9 or TLR4+MD2, they responded to the appropriate TLR ligands and activated the NF- $\mathrm{KB}$ reporter gene (Figure 3 ). In contrast, HEK cells expressing TLR1-11 failed to respond to MSU crystals (Figure 3). Although we did not have natural ligands for TLR10 and TLR11,

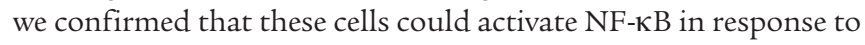
IL-1 $\beta$. Thus, MSU does not stimulate HEK cells through any of the 11 TLRs. Taken together, the results of the loss-of-function and gain-of-function experiments suggest that MSU does not stimulate the inflammatory response through any of the known TLRs. These data also indicate that our MSU crystal preparations lacked contaminating TLR agonists.

The role of TIR adaptor proteins in MSU-stimulated inflammation. TLRs signal through intracellular TIR adaptor molecules (30). In a related set of experiments, we examined whether MSU stimulated 
A

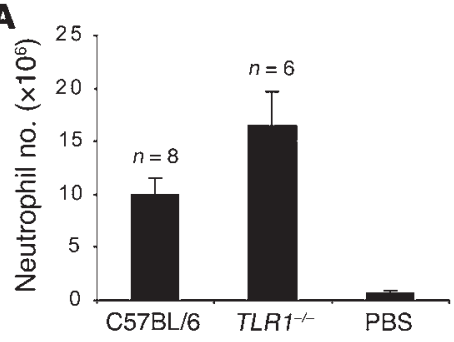

D

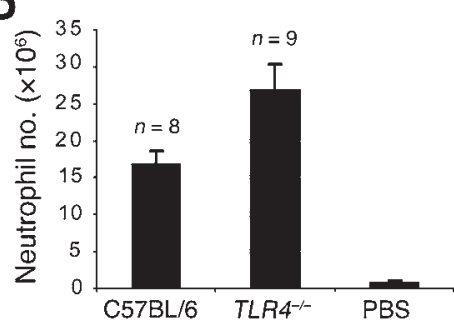

G

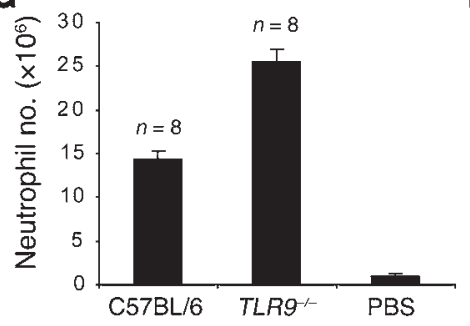

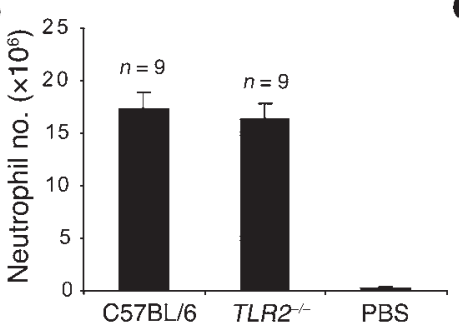

E

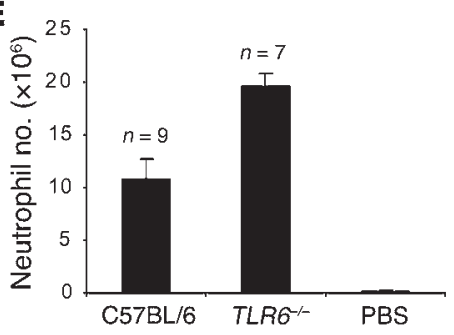

H

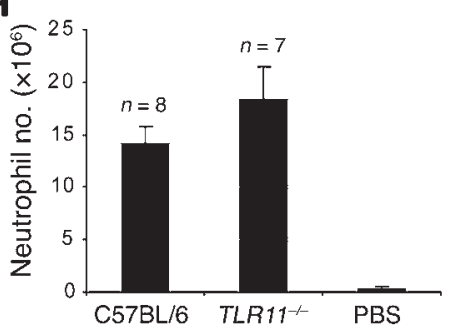

c

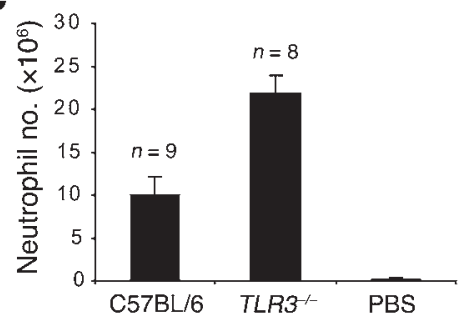

F

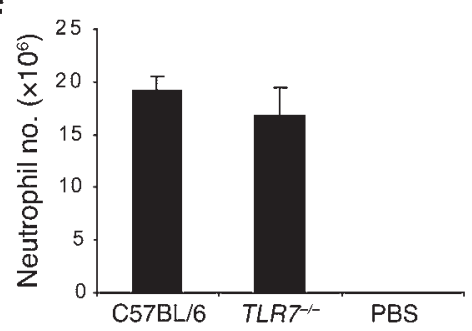

I

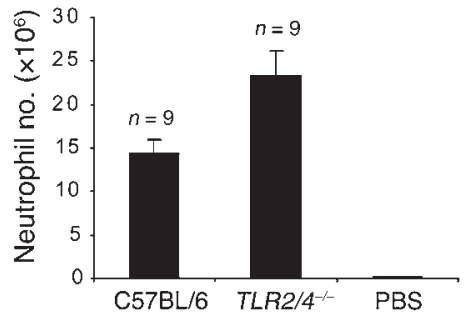

Figure 2

MSU-induced inflammation does not require TLR1, -2, -3, -4, -6, -7, -9, and -11. C57BL/6 mice and mice deficient for TLR1 (A), TLR2 (B), TLR3 (C), TLR4 (D), TLR6 (E), TLR7 (F), TLR9 (G), TLR11 (H), or TLR2/4 (I) were challenged i.p. with 3 mg of MSU crystals (in PBS). C57BL/6 mice challenged with PBS served as negative controls. At 16 hours after challenge, neutrophil numbers in PECs were determined. Data (mean \pm SEM) shown in A-E and G-I were combined from 3 independent experiments, and $n$ 's reported represent the combined numbers of animals for each group. Data shown in $\mathbf{F}$ are from a single experiment due to the limited availability of animals $(n=3)$.

inflammation in mice deficient for each of the 4 known TIR adaptors: MyD88, TIR domain-containing adaptor protein/MyD88 adaptor-like protein (TIRAP/Mal), TIR domain-containing adaptor-inducing IFN- $\beta$ (TRIF), and TRIF-related adaptor molecule (TRAM). There was no reduction in inflammation in mice deficient for TRAM (Figure 4A), TIRAP/Mal (Figure 4B), or TRIF (Figure 4C). Surprisingly however, the acute inflammatory response in MyD88-deficient mice was reduced by $92 \%(P=0.001)$ at 6 hours and by $93 \%(P=0.00013)$ at 16 hours (Figure $4 \mathrm{D})$. We also assayed the peritoneal lavage fluid for the presence of KC and MIP-2, which are 2 chemokines that attract neutrophils. MSU induced high levels of KC and MIP-2 in the peritoneal cavity of WT mice. Consistent with the reduced neutrophil infiltration, in MyD88deficient mice the production of KC and MIP-2 was reduced by $90 \%$ and $77 \%$, respectively (Figure 4, E and F).

Since MSU crystals failed to stimulate through any of the TLRs, it seemed unlikely that the MyD88-dependent inflammation was due to any microbial contaminants in our crystals preparations. However, to rule out this possibility, we treated the MSU crystals with uricase, a highly specific enzyme that oxidizes uric acid; this treatment eliminates MSU but would have no effect on a microbial contaminant. Uricase treatment of MSU crystals destroyed their ability to induce acute inflammation (Figure 1C). Therefore, these results demonstrate that MSU crystals stimulate acute inflamma- tion in vivo through a MyD88-dependent pathway and one that does not appear to be utilizing TLRs.

Role of $I L-1$ and $I L-18$ receptors in MSU-stimulated inflammation. The other receptors that are well known to signal through MyD88 are the IL-1 receptor (IL-1R) and IL-18R. Given the key role of MyD88 in gouty inflammation, we sought to evaluate the role of these 2 cytokine receptors in this response. MSU crystals were injected into the peritoneal cavity of IL-1R or IL-18R-deficient mice, and the acute inflammatory response was evaluated. There was a reduction of $85 \%$ in the neutrophil response in IL-1R mutant mice at both 6 hours and 16 hours (Figure 5A). Similarly, the levels of neutrophil chemoattractants KC and MIP-2 were also reduced in IL-1R mutant mice (Figure 5, B and C). In contrast, the inflammatory response was not reduced in IL-18R-deficient mice (Figure $5 \mathrm{D})$. These results demonstrated that the IL-1R plays an important role in the acute inflammatory response to MSU.

We next sought to determine precisely how the IL-1R was participating in the inflammatory response to MSU. The identity of the cellular receptor(s) that are stimulated by MSU crystals is not clear. Our findings raised the possibility that the IL-1R might be the cellular receptor for MSU. To evaluate this possibility, we tested the ability of MSU crystals to stimulate leukocytes from WT and IL-1R-deficient mice. We found that when WT peritoneal resident cells (primarily macrophages) were stimulated ex vivo with MSU, 

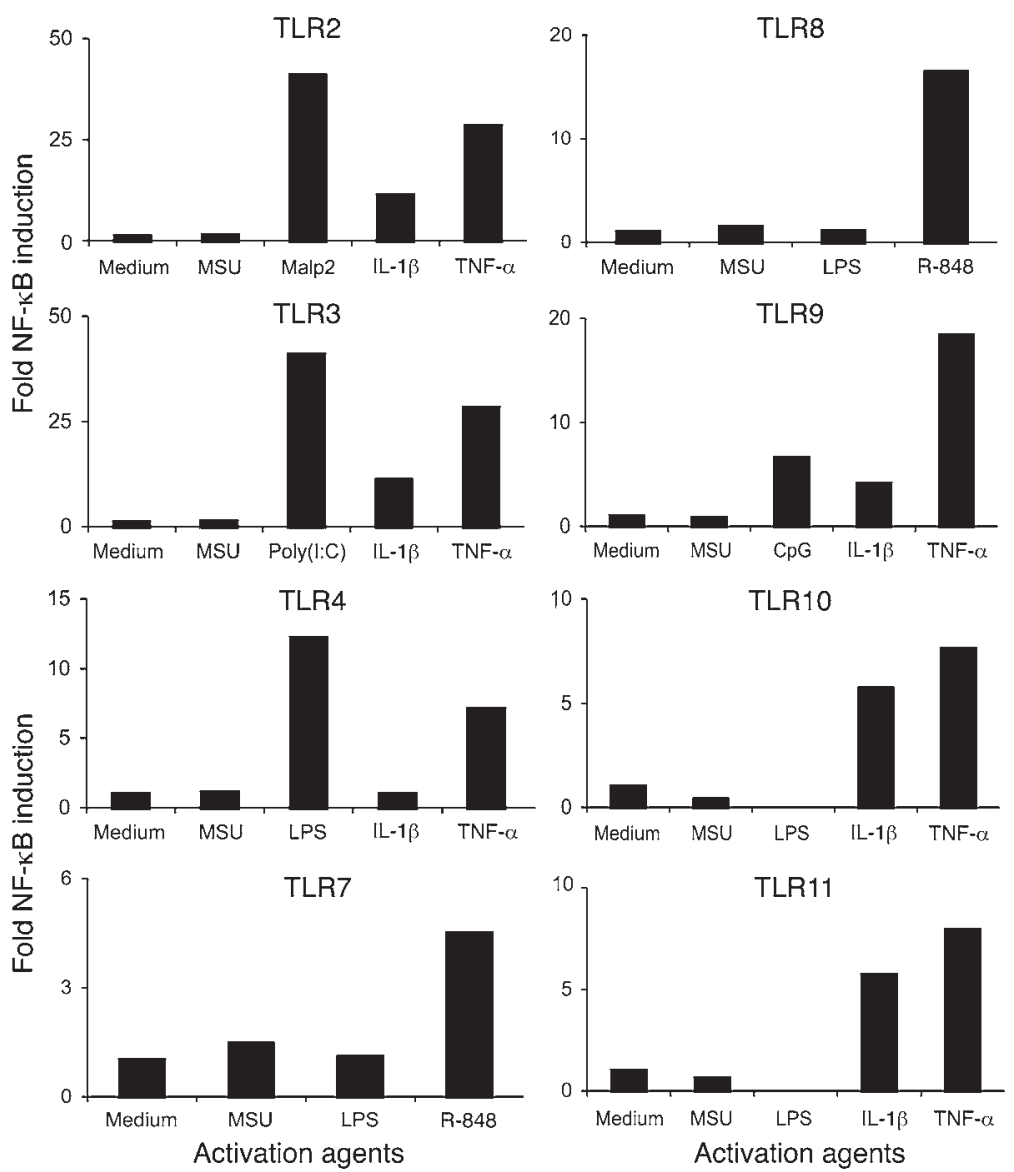

Figure 3

MSU does not stimulate transfected TLRs in vitro. HEK cells were prepared as described in Methods. The following TLR ligands were added to the cells: Malp2 (100 ng/ml), Poly(l:C) $(200 \mu \mathrm{g} / \mathrm{ml})$, LPS (1 ng/ml), R-848 $(1 \mu \mathrm{g} / \mathrm{ml})$, and $\mathrm{CpG}(5 \mu \mathrm{g} / \mathrm{ml})$. TLR10 and TLR11 ligands were unavailable for the test. The readout is the fold increase of firefly luciferase/Renilla luciferase ratio over the unstimulated control. Data shown are representative of 2 or more experiments.

optimal IL-1 $\beta$ production via autocrine or paracrine mechanisms that amplify the response.

Analysis of where the IL-1R-MyD88 pathway functions in vivo. The IL-1R is broadly expressed, and many cell types can produce IL-1. To further understand where the IL-1R-MyD88 pathway was needed in MSU responses, we analyzed mice chimeric for the IL-1R or MyD88. WT mice that were reconstituted with IL-1R-deficient bone marrow $\left(I L-1 R^{-/} \rightarrow \mathrm{WT}\right)$ showed no significant reduction in inflammatory responses to MSU crystals in 7 of 8 experiments that we performed (Figure 8A); in one experiment, the chimeric mice showed a significantly reduced neutrophil recruitment. Overall, there was no significant difference in inflammation between the IL-1R $\left(I L-1 R^{-/-} \rightarrow\right.$ WT $)$ and WT $(\mathrm{WT} \rightarrow \mathrm{WT})$ chimeric mice. Therefore, the IL-1R is not required on leukocytes for this response. This result was consistent with our observation that MSU stimulated IL- $1 \beta$ production in IL-1R-deficient peritoneal cells (Figure 6A). In contrast, IL-1R-deficient mice reconstituted with WT bone marrow $\left(\mathrm{WT} \rightarrow I L-1 R^{-/-}\right.$) had markedly reduced inflammatory responses to MSU crystals (Figure 8B). Therefore,

they produced as expected the proinflammatory cytokine IL-1 $\beta$ (Figure 6A) as well as TNF- $\alpha$ (Figure 6B), albeit at lower levels. Importantly, these responses were not reduced in peritoneal cells from IL-1R-deficient mice. Therefore, the IL-1R is not required for these cells to respond to MSU crystals.

Another obvious possibility was that the IL-1R was needed to respond to IL-1 that was produced in response to MSU. To test this possibility, we injected MSU crystals into mice that were treated with neutralizing antibodies to IL-1 $\alpha$ and IL-1 $\beta$. The anti-IL-1 antibodies caused a significant reduction in the inflammatory response (Figure $7 \mathrm{~A}$ ). This reduction in acute inflammation was not as great as that observed in IL-1R-deficient mice, presumably because the antibody treatment was not neutralizing IL-1 completely. We conclude that MSU crystals stimulate the production of IL- 1 that then binds the IL-1R and signals through MyD88 to cause inflammation.

Given the key role of the IL-1R-MyD88 pathway in the MSU response, we further examined the generation of IL-1 $\beta$ in this model. We measured the levels of IL- $1 \beta$ in the peritoneal fluids and found that MSU stimulated the production of IL-1 $\beta$ in WT mice, but IL-1 $\beta$ production was significantly reduced in both MyD88-deficient and IL-1R-deficient mice (Figure 7B). These results indicate that the IL-1R-MyD88 pathway plays a role in the optimal production of IL- $1 \beta$ in vivo, possibly because this pathway is required for the efficient recruitment of neutrophils and monocytes, which then can be stimulated by MSU to produce IL-1 $\beta$. Alternatively, the IL-1R-MyD88 pathway might be required for the IL-1R is required on radioresistant (non-bone marrow-derived) host cells to respond to IL-1. Consistent with this result, our preliminary data indicate that MSU-stimulated inflammation was reduced in MyD88-deficient mice reconstituted with WT bone marrow (data not shown). In the chimeric mice whose bone marrow-derived but not other cells lacked MyD88 (MyD88-/- $\rightarrow$ WT $)$, MSU-stimulated inflammatory response was significantly reduced in only 1 of 8 experiments. These experiments overall showed no significant reduction in inflammation in $M y D 88^{-/-} \rightarrow$ WT mice (Figure 8A), indicating that MyD88 expression in bone marrow-derived cells is not essential. Therefore, MyD88 is required in the radioresistant host cells, presumably for its role in transducing IL-1R signals.

\section{Discussion}

One of the central observations in this report is that MSU crystals do not stimulate acute inflammation in mice that genetically lack the TIR adaptor protein MyD88. Mice lacking MyD88 showed reduced production of the proinflammatory cytokines IL-1 $\beta, \mathrm{KC}$, and MIP-2 and showed reduced neutrophil accumulation in response to MSU in vivo. Therefore, our data identify MyD88 as a key signaling molecule in MSU-stimulated inflammation. In contrast, MSU did stimulate inflammation in mice that genetically lacked TIRAP/Mal, TRIF, or TRAM. Therefore, among the TIR adaptors, MyD88 plays a unique role in MSU-stimulated inflammation.

Another major finding is that MyD88 is primarily required for transducing signals downstream of the IL-1R, while most, and probably all, TLRs and the IL-18R are not essential for the inflam- 

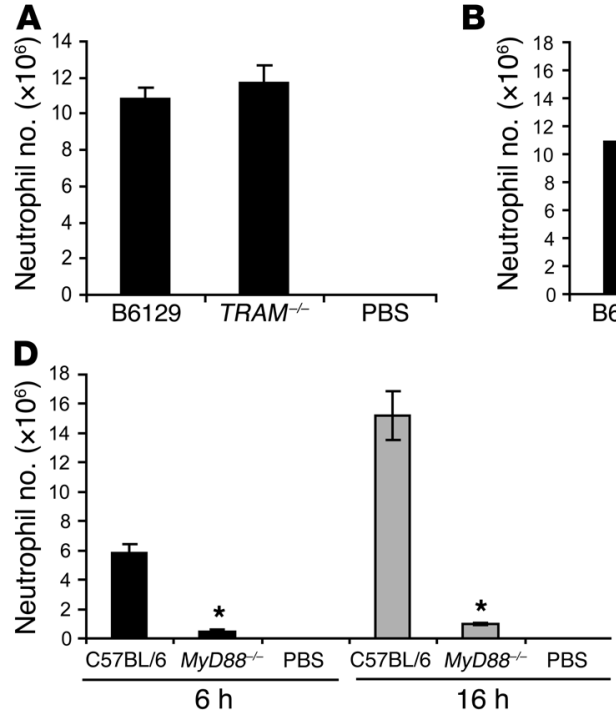

B

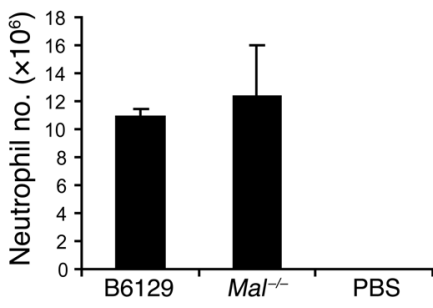

E

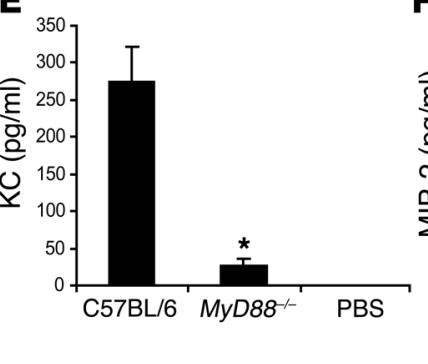

C

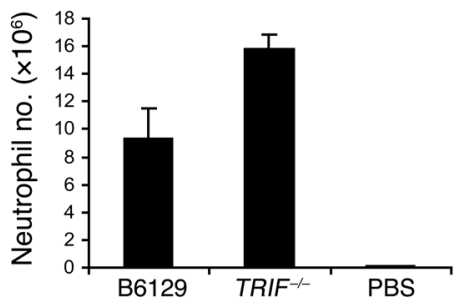

$\mathbf{F}$

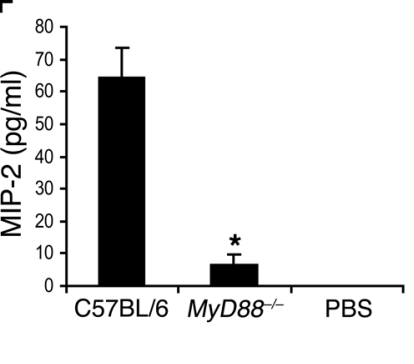

Figure 4

MyD88, but not TRAM, Mal, or TRIF, is required for MSU-induced KC and MIP-2 production and neutrophil infiltration in the peritoneal cavity. (A-C) B6129 mice and mice deficient for TRAM (A), Mal (B), or TRIF (C) were challenged i.p. with 3 mg of MSU crystals (in PBS). B6129 mice challenged with PBS served as negative controls. At 16 hours after MSU challenge, neutrophil numbers in the PECs were determined $(n=3)$. (D-F) C57BL/6 and MyD88-/- mice were challenged i.p. with $3 \mathrm{mg}$ of MSU crystals (in PBS). C57BL/6 mice challenged with PBS served as negative controls. (D) At the indicated time points, neutrophil numbers in the PECs were determined ( $n=3$ for 6 hours and $n=4$ for 16 hours). At 6 hours after MSU challenge, KC (E) and MIP-2 (F) concentrations in the peritoneal lavage fluids were determined by ELISA $(n=3)$. Data shown are representative of 3 or more experiments. ${ }^{*} P<0.01$ versus control (C57BL/6) in $\mathbf{D}-\mathbf{F}$.

matory response to MSU. Our results clearly demonstrate that IL-1 is a major proinflammatory cytokine mediating the neutrophilic gouty inflammation to MSU in vivo. While IL-1 is known to be a proinflammatory cytokine and produced in response to MSU, its key role in gouty inflammation in vivo has not, to our knowledge, previously been shown. Experiments with chimeric mice further demonstrated that this IL-1R-MyD88-dependent proinflammatory pathway is operating mainly in non-bone marrow-derived cellular elements.
Whether MSU stimulates a specific cell surface receptor(s) on tissue-resident or -lining cells to produce proinflammatory cytokines and initiate the inflammatory response is unclear. Our finding that MSU-stimulated inflammation requires MyD88 raised the possibility that TLR signaling played some role in mediating the inflammatory response. However, MSU-stimulated inflammation was not affected in the mice genetically deficient in TLR1, $-2,-4$, $-2 / 4,-6,-7,-9$, or -11 , ruling out the possibility that any of these MyD88-dependent TLRs is essential for the response. TLR3, which

\section{Figure 5}

MSU-induced inflammation is IL-1R dependent and IL-18R independent. C57BL/6 mice and mice deficient for IL-1R (A-C) or IL-18R (D) were challenged i.p. with $3 \mathrm{mg}$ of MSU crystals (in PBS). C57BL/6 mice challenged with PBS served as negative controls. (A) At indicated time points, neutrophil numbers in the PECs were determined $(n=4)$. At 6 hours after MSU challenge, KC (B) and MIP-2 (C) concentrations in the peritoneal lavage fluids were determined by ELISA $(n=4)$. (D) At 16 hours after MSU challenge, neutrophil numbers in the PECs were determined $(n=4)$. Data shown in $\mathbf{A}-\mathbf{C}$ are representative of 3 or more experiments. Data shown in $\mathbf{D}$ are representative of 2 experiments. ${ }^{*} P<0.01$ versus control (C57BL/6) in A and $\mathbf{B}$.
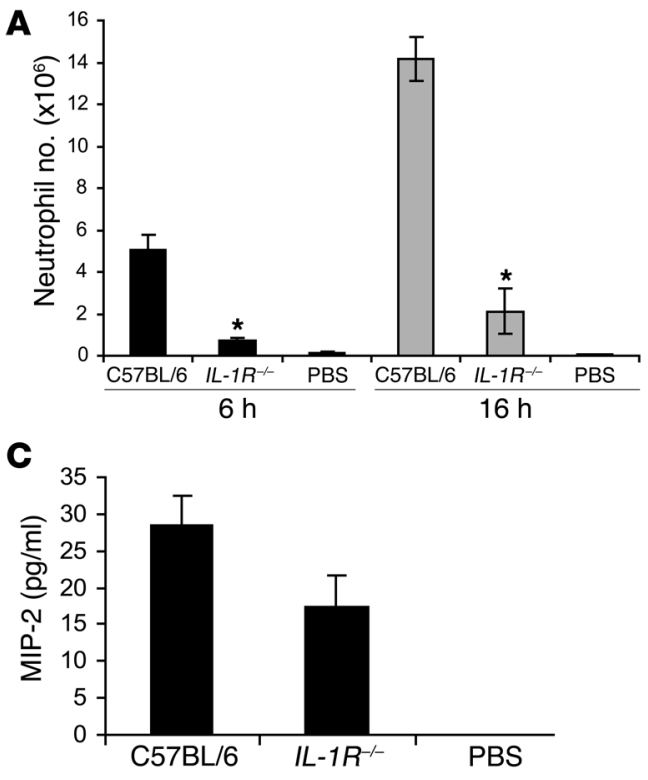

B
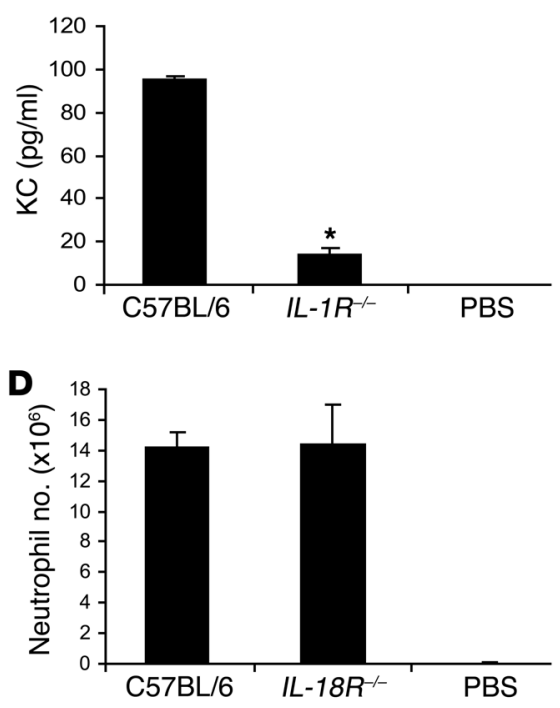
A

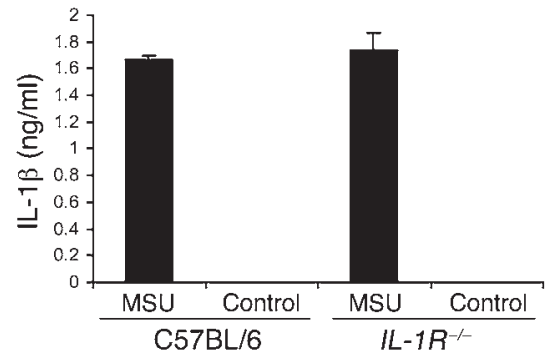

B

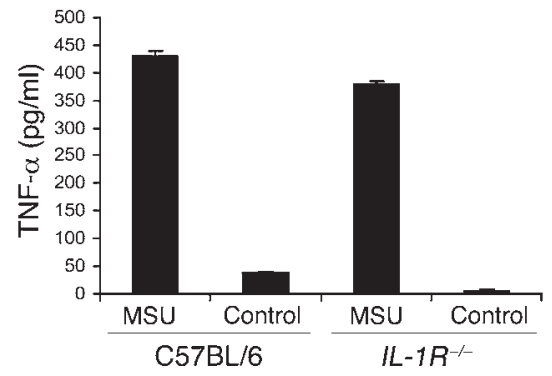

signals through TRIF instead of MyD88, was also not involved. Furthermore, the finding that MSU fails to stimulate HEK cells expressing TLR1-11 also makes it unlikely that MSU directly stimulates any TLRs. While this report was in preparation, Liu-Bryan et al. reported that MSU-stimulated inflammatory response was attenuated in $M y D 88^{-/-}$mice; however, in contrast to our results, they found that the inflammation was also reduced in mice lacking the MyD88-associated receptors TLR2 and TLR4 (34). The discrepancies between their and our results may be due to the different in vivo models employed; Liu-Bryan et al. injected MSU into subcutaneous air pouches, while we injected MSU into the peritoneal cavity; perhaps cells in different tissues respond to MSU stimulation through different mechanisms. Alternatively, perhaps Liu-Bryan's crystal preparations differ in other ways that allowed them to interact with TLRs.

Although we can find no evidence that in vivo MSU is working through TLRs, it remains formally possible that MSU-stimulated inflammation may involve several TLRs that are activated redundantly or some TLR(s) that we have not examined in vivo, e.g., TLR5 or TLR8. This possibility is made unlikely by our finding that MSU does not stimulate HEK cells expressing TLR1-11; however, we cannot exclude the possibility that HEK cells lack the expression of some molecules that are required to form complexes with certain TLRs, which may mediate the response to

\section{Figure 7}

The role of IL-1 in MSU-induced inflammation. (A) C57BL/6 mice were administered i.v. with anti-IL-1 antibodies or PBS (vehicle). Thirty minutes later, the treated mice and $I L-1 R^{-1-}$ mice were challenged i.p. with MSU crystals. C57BL/6 mice challenged with PBS served as negative controls. At 6 hours after MSU challenge, neutrophil numbers in the PECs were determined $(n=4)$. ${ }^{*} P<0.01$ versus control (vehicle). (B) C57BL/6, MyD88 ${ }^{-/}$, and $I L-1 R^{-/-}$mice were challenged i.p. with $3 \mathrm{mg}$ of MSU crystals (in PBS). C57BL/6 mice challenged with PBS served as negative controls. At 6 hours after MSU challenge, IL-1 $\beta$ concentration in the peritoneal lavage fluids were determined by ELISA $(n=5)$. Data shown are representative of 2 experiments in $\mathbf{A}$ and 3 experiments in B. ${ }^{*} P<0.01$ versus control (C57BL/6).

\section{Figure 6}

IL-1R is not the receptor for MSU stimulation. Peritoneal resident cells from C57BL/6 and $I L-1 R^{-/-}$mice were harvested and stimulated with or without MSU crystals as described in Methods. At 16 hours after MSU stimulation, IL-1 $\beta$ (A) and TNF- $\alpha(B)$ concentrations in the culture fluids were determined by ELISA $(n=2)$. Data shown are representative of 3 or more experiments.

MSU stimulation. This possibility could explain the different observations made by us and by Liu-Bryan et al., who reported that TLR2 expression in chondrocytes plays a role in MSUinduced nitric oxide production (38). However, we do not favor the possibility that TLR2 plays an essential role in vivo, because in our system, mice lacking TLR2, or both TLR2 and TLR4 together, show an undiminished inflammatory response to MSU crystals. Interestingly, in mice lacking TLR4, we observed a significant increase in neutrophil infiltration. It is possible that this is because TLR 4 mediates some antiinflammatory function, such as TGF- $\beta 1$ production from differentiated macrophages, during MSU-induced inflammation $(34,39)$. However, the basis for the observation in our studies was not elucidated, and other potential mechanisms are possible.

We also show that MSU does not signal directly through the IL-1R or IL-18R, both of which are MyD88-dependent receptors. Other cell surface receptors that have been proposed to respond to MSU include the CD16 and complement receptors on neutrophils (40) and membrane integrins (GPIIb/IIIa) on platelets (41). However, these in vitro studies do not provide information as to whether these receptors are essential for MSU-stimulated inflammation in vivo. In fact, we have been able to stimulate human monocytic leukemia THP-1 cells, which lack the expression of CD16 (42), with MSU to produce IL-1 $\beta$ (unpublished observations). Furthermore, MSU-stimulated dendritic cell activation cannot be blocked by an anti-CD16 antibody (unpublished observations).

Instead, our data indicate that MSU stimulates cells to produce IL-1 (through as-yet-unknown mechanism[s]/receptor[s]). The IL-1 that is produced is essential for gouty inflammation, as neutralizing it with antibodies suppressed the inflammatory response.
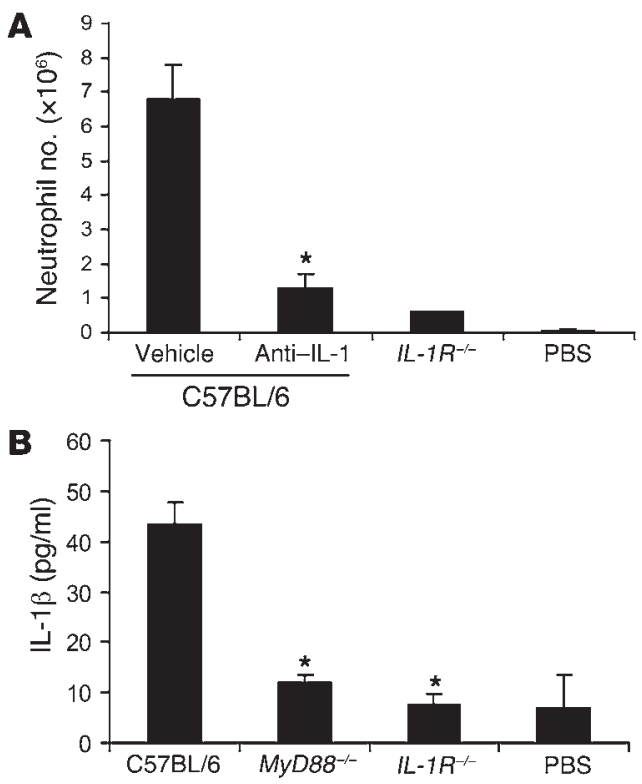
A

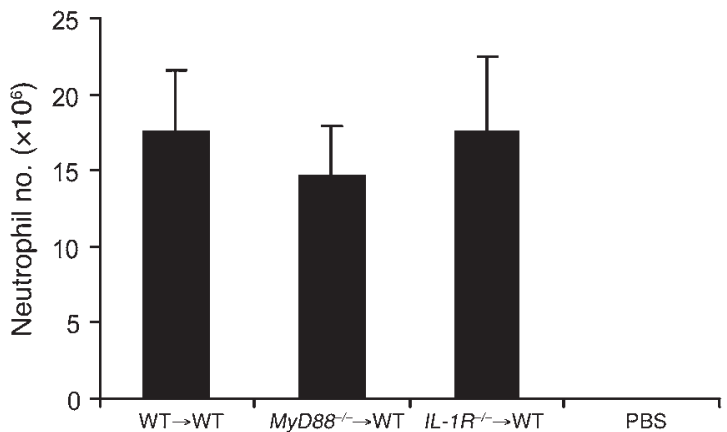

B

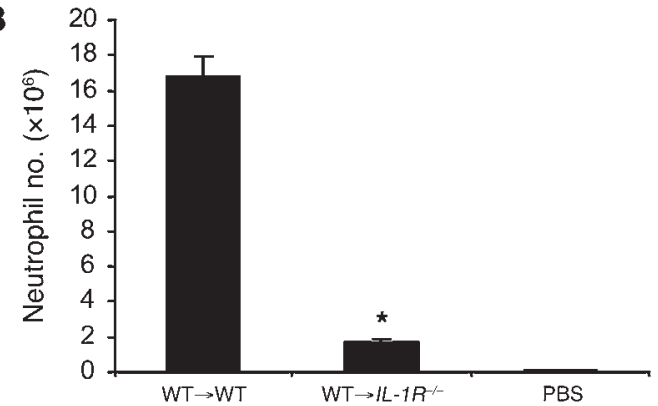

Moreover, mice deficient in the IL-1R showed markedly reduced inflammatory responses. Although the IL-1R is not involved in MSU triggering of cells to initially produce IL-1, our data indicate that the resulting IL-1 stimulation of the IL-1R amplifies the production of proinflammatory cytokines through a MyD88-dependent pathway and is a major step in controlling the development of full-blown inflammation. While it has been recognized that MSU stimulates cells to produce IL-1 (15-17) along with many other cytokines, the central role of IL-1 in gouty inflammation has not been previously shown. While this report was under review, Martinon and colleagues reported that MSU crystals engage the caspase-1-activating NALP3 inflammasome, resulting in the production of active IL-1 $\beta$ and IL-18 (43). Their studies provide the mechanistic evidence of IL-1 production in response to MSU stimulation; furthermore, they also show that MSU-induced inflammation is impaired in mice deficient in inflammasome or IL-1R, consistent with our findings that IL-1 production and IL-1R signaling play essential roles in MSU-induced inflammation.

Chimeric mice whose radioresistant cells lack the IL-1R or MyD88 showed markedly reduced inflammatory responses similar to those in $I L-1 R^{-/-}$or $M y D 88^{-/-}$mice, indicating that expression of IL-1R or MyD88 in bone marrow-derived cells alone is not sufficient for MSU to stimulate inflammation. In contrast, MSU-stimulated inflammatory responses were not reduced in chimeric mice lacking IL-1R or MyD88 only on bone marrow elements. Therefore, the IL-1R-MyD88-dependent step is mainly operating in non-bone marrow-derived cells. We speculate that these non-hematopoietic cells include vascular endothelium, as IL-1 activates endothelial cells and induces the expression of the leukocyte adhesion molecule E-selectin (44). Both IL-1 and TNF- $\alpha$ are major proinflammatory cytokines that activate endothelial cells (44). When peritoneal resident cells were stimulated with MSU ex vivo, more (>3-fold) IL-1 $\beta$ than TNF- $\alpha$ was produced, and when mice were challenged with MSU in vivo, the level of TNF- $\alpha$ in the lavage fluid at 6 hours was undetectable,

\section{Figure 8}

Functions of IL-1R and MyD88 in bone marrow- versus non-bone marrow-derived cells mediating MSU-stimulated inflammation. Bone marrow chimeras were generated as described in Methods. (A) C57BL/6 (WT) mice served as hosts, and B6.SJL (WT), MyD88-/-, or $I L-1 R^{-/-}$mice served as bone marrow donors. (B) C57BL/6 (WT) and $I L-1 R^{-1-}$ mice served as hosts, and B6.SJL (WT) mice served as bone marrow donors. (A and $\mathbf{B}$ ) After bone marrow reconstitution, chimeric mice were challenged i.p. with $3 \mathrm{mg}$ of MSU crystals (in PBS). C57BL/6 mice challenged with PBS served as negative controls. At 16 hours after MSU challenge, neutrophil numbers in the PECs were determined $(n=3)$. Data shown in $\mathbf{A}$ are representative of 8 experiments; data shown in $\mathbf{B}$ are representative of 3 experiments. ${ }^{*} P<0.01$ versus control $(\mathrm{WT} \rightarrow \mathrm{WT})$.

presumably because it was produced at levels below the limit of detection in our assay (data not shown). Since much more IL- 1 than TNF- $\alpha$ was produced in response to MSU, IL-1 may be responsible for vascular endothelial activation. Also consistent with this speculation is that endothelial cells are primarily responsible for producing neutrophil-attracting chemokine KC (45), and the MSU-stimulated production of this chemokine was markedly reduced in $I L-1 R^{-/-}$and $M y D 88^{-/-}$mice. KC is thought to be important to the MSU-induced acute inflammatory responses because the neutrophil influx is inhibited in mice lacking the receptor for KC (CXCR2) (46). In the absence of endothelial activation and high-level expression of selectins and $\mathrm{KC}$, neutrophil adhesion to the endothelium and subsequent extravasation would be markedly reduced. IL-1 will also affect other non-hematopoietic cells, and these may also contribute to the inflammatory process.

Our data lead to the model shown in Figure 9. In vivo, nucleation of MSU converts the nonphlogistic form of uric acid to MSU crystals. This chemical phase change allows the MSU to then interact with cells in ways that stimulate the production of IL-1 and other cytokines. The IL-1 that is produced then stimulates the IL-1R on non-bone marrow-derived cells, resulting

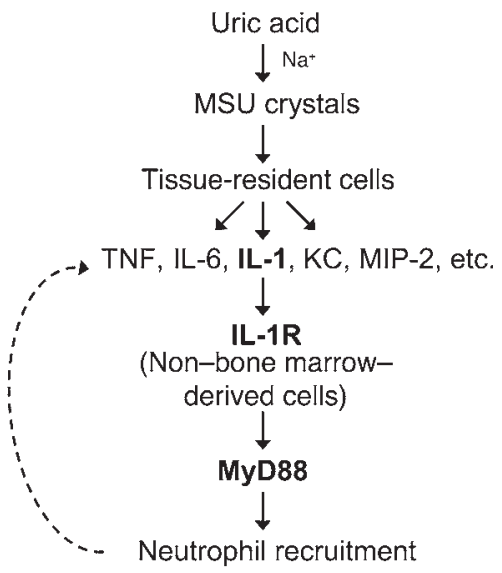

Figure 9

Proposed mechanism for MSU-induced inflammation. When uric acid nucleates to form MSU crystals, tissue resident cells are stimulated to produce IL-1 and other cytokines. The IL-1 that is produced then stimulates the IL-1R on non-bone marrow-derived cells, resulting in a MyD88-dependent amplification of the proinflammatory response. 
in a MyD88-dependent amplification of the proinflammatory response (including the production of more $\mathrm{IL}-1$ and chemokines that attract neutrophils [e.g., KC and MIP-2]) and the recruitment of neutrophils. Together, these findings provide important new insights into gout, a disease recognized since the time of antiquity but still incompletely understood. The data clearly demonstrate that a cellular adaptor protein, MyD88, is necessary for the MSU-stimulated inflammatory response. In addition, our findings demonstrate a central role for IL-1 signaling through the IL-1R-MyD88 pathway in gouty inflammation.

\section{Methods}

Animals and cell lines. C57BL/6, IL-1 $R^{-/-}$, and $I L-18 R^{-/-}$mice (8-12 weeks old) were purchased from the Jackson Laboratory. B6 × $129 \mathrm{~F}_{1}(\mathrm{~B} 6129)$ mice (8-12 weeks old) were purchased from Taconic. MyD88-/- (47), TIRAP/Mal ${ }^{-/}$(48), TRIF ${ }^{-/-}$(49), TRAM ${ }^{-/-}$(50), TLR1 $1^{-/-}$(51), TLR2 $2^{-/-}(52)$,

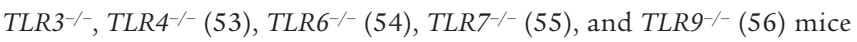
were generated at the Department of Host Defense, Osaka University, Osaka, Japan. TLR11-/- (57) mice were kindly provided by Sanker Ghosh (Yale University, New Haven, Connecticut, USA). TLR2/4-/- mice were generated by crossing TLR2 ${ }^{-/-}$with $T L R 4^{--}$mice. All animal studies were approved by the Institute Animal Care and Use Committee of the University of Massachusetts Medical School, and all mice were kept in the University of Massachusetts Medical School animal facilities. HEK 293 cells were maintained in hybridoma culture medium (HCM) consisting of RPMI 1640 plus 10\% FBS, 2 mM L-glutamine, 10 mM HEPES, penicillin/streptomycin, and $50 \mu \mathrm{M} 2$-mercaptoethanol.

Reagents. Poly(I:C) was purchased from Amersham Biosciences. LPS and uric acid were purchased from Sigma-Aldrich. CpG (ODN1826) was purchased from Coley. R-848 was purchased from GLSynthesis Inc. Malp2 was purchased from EMC Microcollections. Uricase (Elitek) was purchased from Sanofi-Aventis. MSU crystals were prepared by first dissolving uric acid $(5 \mathrm{mg} / \mathrm{ml})$ in $0.1 \mathrm{M}$ borate buffer ( $\mathrm{pH} 8.5)$, and, after passing through a $0.45-\mu \mathrm{m}$ filter, the supersaturated uric acid solution was left at room temperature for 48 hours. The large crystals formed during the first 48 hours were removed by centrifugation, and the supernatant uric acid solution was incubated at room temperature for 5 more days to allow the formation of fine crystals, which were harvested by centrifugation. After 2 washes with absolute alcohol and 1 wash with acetone, the crystals were allowed to air dry. Crystal size varied between $3 \mu \mathrm{m}$ and $50 \mu \mathrm{m}$ in length, with $48 \%$ of crystals less than $10 \mu \mathrm{m}$ in length and a mean \pm SD crystal size of $11.69 \pm 6.6 \mu \mathrm{m}$. All reagents were prepared under pyrogen-free conditions.

Luciferase reporter assay. HEK 293 cells were grown to about $80 \%$ confluence in a 96-well plate and transfected with $80 \mathrm{ng} \mathrm{NF- \kappa B} \mathrm{luciferase} \mathrm{report}$ gene and $40 \mathrm{ng}$ of control Renilla luciferase reporter gene, along with $3 \mu \mathrm{g}$ of various constructs expressing mouse TLR2, $-3,-4,-7,-9$, or -11 or human TLR8 or TLR10 (all in PCDNA3 vector) $(58,59)$. Sixteen hours later, cells were washed once with fresh medium and added with $1 \mathrm{ng} / \mathrm{ml}$ of recombinant human IL- $1 \beta$ or TNF- $\alpha$ (R\&D Systems), $300 \mu \mathrm{g} / \mathrm{ml}$ of MSU crystals, or TLR ligands, as indicated in the figure legends, in a total volume of 100 $\mu \mathrm{l}$ per well. Luminescence was read 6 hours later using the Dual-Luciferase Reporter Assay System (Promega) and a fluorometer.

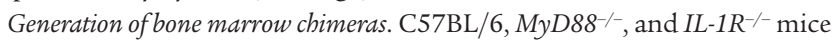
were lethally irradiated using $11 \mathrm{~Gy}$. Bone marrow was prepared from the femurs and tibias of B6.SJL-Ptprca/BoAiTac (B6.SJL, CD45.1), MyD88-/-, or $I L-1 R^{-1-}$ donor mice and depleted of T cells using a mAb against Thy1, M5/149 (ATCC), and complement (Pel-Freez Biologicals). The irradiated mice were reconstituted i.v. with $2.5 \times 10^{6}$ of different $\mathrm{T}$ cell-depleted bone marrow cells, as indicated. The mice were then housed for 3-4 months to allow for the turnover and reconstitution of bone marrow-derived cells.
The reconstitution of the chimeras was confirmed by staining blood leukocytes with anti-CD45.1 and anti-CD45.2 antibodies (BD Biosciences) and flow cytometric analysis.

MSU-induced inflammation in vivo. Mice were injected i.p. with $3 \mathrm{mg}$ MSU crystals in $0.1 \mathrm{ml} \mathrm{PBS}, 1.5 \mathrm{mg}$ MSU in $0.3 \mathrm{ml}$ borate buffer (0.1 M, pH 8.5), or $1.5 \mathrm{mg}$ uricase-digested MSU (in $0.3 \mathrm{ml}$ borate buffer). Equal volumes of PBS or borate buffer were injected into 2 mice and served as negative controls. The TLR-deficient mice were originally on the 129 background, and some were not yet fully backcrossed onto the C57BL/ 6 background. In preliminary experiments, we found that the response of C57BL/ 6 and B6129 $\mathrm{F}_{1}$ mice to MSU is not different (data not shown) and therefore used C57BL/ 6 mice for a comparison. To analyze the involvement of IL-1 in MSU-induced inflammation, mice were administered i.v. with $10 \mu \mathrm{g}$ each of neutralizing rabbit polyclonal anti-IL-1 $\alpha$ and anti-IL-1 $\beta$ antibodies (in $0.3 \mathrm{ml}$ PBS) (Abcam) or $0.3 \mathrm{ml}$ PBS 30 minutes before MSU injection. At different time points, animals were euthanized by $\mathrm{CO}_{2}$ exposure, and their peritoneal cavities were washed with $6 \mathrm{ml} \mathrm{HCM} \mathrm{containing}$ $3 \mathrm{mM}$ EDTA and $10 \mathrm{U} / \mathrm{ml}$ heparin. Total numbers of peritoneal exudate cells (PECs) were counted by a hematocytometer, and lavage fluids were centrifuged at $450 \mathrm{~g}$ for 10 minutes. Supernatants were stored at $-20^{\circ} \mathrm{C}$ before analysis for cytokines, and cells were resuspended in HCM and subjected to staining and flow cytometric analysis. Neutrophil numbers in the PECs were determined by multiplying the total cell numbers by the percentage of $\mathrm{Ly}-6 \mathrm{G}^{+} 7 / 4^{+}$cells.

Flow cytometric analysis. PECs $\left(1 \times 10^{6}\right)$ were incubated with mAb $2.4 \mathrm{G} 2$ for 30 minutes to block FcyRIB/III receptors and stained with mAbs Ly-6G-FITC (BD Biosciences) and 7/4-biotin (AbD Serotec) for $30 \mathrm{~min}$ utes at $4^{\circ} \mathrm{C}$. The cells were further incubated with streptavidin-APC. Following staining, cells were washed with PBS, fixed in PBS containing $2 \%$ paraformaldehyde, and analyzed on a FACSCalibur (BD Biosciences). Data were acquired by CellQuest software (BD Biosciences) and analyzed by FlowJo software (Tree Star Inc.).

Cytokine production analysis. Peritoneal resident cells from C57BL/6 and $I L-1 R^{-/-}$mice were harvested by lavage. Cells were washed once with $\mathrm{HCM}$ and resuspended in HCM at a density of $3 \times 10^{6} / \mathrm{ml}$, and $0.5 \mathrm{ml}$ of the cell suspension was plated into a 24-well plate. Cells were treated with MSU crystals $(0.5 \mathrm{mg} / \mathrm{ml})$ or left untreated. At 16 hours after treatment, IL-1 $\beta$ and TNF- $\alpha$ concentrations in the culture medium were determined by ELISA according to the manufacturer's instructions (R\&D Systems). KC, MIP-2, IL-1 $\beta$, and TNF- $\alpha$ concentrations in the peritoneal lavage fluids were also determined by ELISA (R\&D Systems).

Statistics. Statistical analysis in each independent experiment was performed with an unpaired, 2-tailed Student's $t$ test. For the experiments shown in Figure 2 that were repeated 3 times, data were combined and analyzed using a linear mixed model (60), with experiment as the random effect. Data in Figure 2 were also analyzed by analysis of variance for mixed models (ANOVAMM) using restricted estimation by maximum likelihood (REML) $(61,62)$ and by pairwise comparisons between WT and TLR-deficient mice by Tukey's honest significant difference using the estimated nondiagonal covariance structure. The distributional characteristics of model residuals were evaluated for normality both graphically by visual inspection of frequency histograms and by the Kolmogorov-Smirnov goodness-of-fit test for normality. The latter analyses were performed using the SAS Proc Mixed statistical program (SAS Institute Inc.). Both analyses of the data in Figure 2 generated concordant results. Data are reported as mean \pm SEM. $P<0.05$ was considered statistically significant.

\section{Acknowledgments}

We thank Evelyn Kurt-Jones and Robert Finberg for advice and mice. We thank Stephen Baker for statistical analysis of data in 
Figure 2. We thank Shelly Galusha for technical assistance. This work was supported by grants from the NIH to K.L. Rock, and core resources supported by Diabetes Endocrinology Research Center grant DK32520 were also used.

Received for publication January 20, 2006, and accepted in revised form June 6, 2006.

1. Terkeltaub, R. 2005. Pathogenesis and treatment of crystal-induced inflammation. In Artbritis and allied conditions. W. Koopman and L.W. Moreland, editors. Lippincott Williams \& Wilkins. Philadelphia, Pennsylvania, USA. 2357.

2. [Anonymous]. 1970. Carl Wilhelm Scheele (17421786) Swedish apothecary. JAMA. 212:2258-2259.

3. Freudweiler, M. 1899. Studies on the nature of gouty tophi. Dtsch. Arch. Klin. Med. 63:266.

4. Rich, A.M., Giedd, K.N., Cristello, P., and Weissmann, G. 1985. Granules are necessary for death of neutrophils after phagocytosis of crystalline monosodium urate. Inflammation. 9:221-232.

5. Tramontini, N., Huber, C., Liu-Bryan, R., Terkeltaub, R.A., and Kilgore, K.S. 2004. Central role of complement membrane attack complex in monosodium urate crystal-induced neutrophilic rabbit knee synovitis. Arthritis Rheum. 50:2633-2639.

6. Hasselbacher, P. 1979. C3 activation by monosodium urate monohydrate and other crystalline material. Arthritis Rheum. 22:571-578.

7. Russell, I.J., Mansen, C., Kolb, L.M., and Kolb, W.P. 1982. Activation of the fifth component of human complement (C5) induced by monosodium urate crystals: C5 convertase assembly on the crystal surface. Clin. Immunol. Immunopathol. 24:239-250.

8. Doherty, M., Whicher, J.T., and Dieppe, P.A. 1983. Activation of the alternative pathway of complement by monosodium urate monohydrate crystals and other inflammatory particles. Ann. Rheum. Dis. 42:285-291.

9. Fields, T.R., Abramson, S.B., Weissmann, G., Kaplan, A.P., and Ghebrehiwet, B. 1983. Activation of the alternative pathway of complement by monosodium urate crystals. Clin. Immunol. Immunopathol. 26:249-257.

10. Webster, M.E., Maling, H.M., Zweig, M.H., Williams, M.A., and Anderson, W., Jr. 1972. Urate crystal induced inflammation in the rat: evidence for the combined actions of kinins, histamine and components of complement. Immunol. Commun. 1:185-198.

11. Abramson, S., Hoffstein, S.T., and Weissmann, G. 1982. Superoxide anion generation by human neutrophils exposed to monosodium urate. Arthritis Rheum. 25:174-180.

12. Jaramillo, M., Naccache, P.H., and Olivier, M. 2004. Monosodium urate crystals synergize with IFN-gamma to generate macrophage nitric oxide: involvement of extracellular signal-regulated kinase 1/2 and NF-kappa B. J. Immunol. 172:5734-5742.

13. Chen, L., et al. 2004. Stimulation of inducible nitric oxide synthase by monosodium urate crystals in macrophages and expression of iNOS in gouty arthritis. Nitric Oxide. 11:228-236.

14. Di Giovine, F.S., Malawista, S.E., Thornton, E., and Duff, G.W. 1991. Urate crystals stimulate production of tumor necrosis factor $\alpha$ from human blood monocytes and synovial cells. Cytokine mRNA and protein kinetics, and cellular distribution. J. Clin. Invest. 87:1375-1381.

15. Roberge, C.J., et al. 1991. Crystal-neutrophil interactions lead to interleukin-1 synthesis. Agents Actions. 34:38-41.

16. Di Giovine, F.S., Malawista, S.E., Nuki, G., and Duff, G.W. 1987. Interleukin 1 (IL 1) as a mediator of crystal arthritis. Stimulation of T cell and synovial fibroblast mitogenesis by urate crystal-induced
Address correspondence to: Kenneth L. Rock, Department of Pathology, University of Massachusetts Medical School, Worcester, Massachusetts 01655, USA. Phone: (508) 856-2521; Fax: (508) 856-1094; E-mail: Kenneth.rock@umassmed.edu.

Yan Shi's present address is: Institute of Infection, Immunity and Inflammation, University of Calgary, Calgary, Alberta, Canada.
IL 1. J. Immunol. 138:3213-3218.

17. Murakami, Y., et al. 2003. Inhibition of monosodium urate monohydrate crystal-induced acute inflammation by retrovirally transfected prostaglandin D synthase. Arthritis Rheum. 48:2931-2941.

18. Guerne, P.A., Terkeltaub, R., Zuraw, B., and Lotz, M. 1989. Inflammatory microcrystals stimulate interleukin- 6 production and secretion by human monocytes and synoviocytes. Arthritis Rheum. 32:1443-1452.

19. Harigai, M., et al. 1993. Monocyte chemoattractant protein-1 (MCP-1) in inflammatory joint diseases and its involvement in the cytokine network of rheumatoid synovium. Clin. Immunol. Immunopathol. 69:83-91.

20. Terkeltaub, R., et al. 1991. Monocyte-derived neutrophil chemotactic factor/interleukin-8 is a potential mediator of crystal-induced inflammation. Arthritis Rheum. 34:894-903.

21. Murakami, Y., Akahoshi, T., Kawai, S., Inoue, M., and Kitasato, H. 2002. Antiinflammatory effect of retrovirally transfected interleukin-10 on monosodium urate monohydrate crystal-induced acute inflammation in murine air pouches. Arthritis Rheum. 46:2504-2513.

22. Ryckman, C., et al. 2003. Role of S100A8 and S100A9 in neutrophil recruitment in response to monosodium urate monohydrate crystals in the air-pouch model of acute gouty arthritis. Arthritis Rheum. 48:2310-2320.

23. Rouleau, P., et al. 2003. The calcium-binding protein S100A12 induces neutrophil adhesion, migration, and release from bone marrow in mouse at concentrations similar to those found in human inflammatory arthritis. Clin. Immunol. 107:46-54.

24. Shi, Y., Evans, J.E., and Rock, K.L. 2003. Molecular identification of a danger signal that alerts the immune system to dying cells. Nature. 425:516-521.

25. Ramon, G. 1925. Sur l'augmentation anormale de l'antitoxine chez les chevaux producteurs de serum antidiptherique. Bull. Soc. Cent. Med. Vet. 101:227-234.

26. Glenny, A.T., Pope, C.G., Waddington, H., and Wallace, U. 1926. Immunology notes. XXIII. The antigenic value of toxoid precipitated by potassium alum. J. Pathol. Bacteriol. 29:31-40.

27. Iwasaki, A., and Medzhitov, R. 2004. Toll-like receptor control of the adaptive immune responses. Nat. Immunol. 5:987-995.

28. Kaisho, T., and Akira, S. 2002. Toll-like receptors as adjuvant receptors. Biochim. Biophys. Acta. 1589:1-13.

29. Matzinger, P. 1998. An innate sense of danger. Semin. Immunol. 10:399-415.

30. Akira, S., and Takeda, K. 2004. Toll-like receptor signalling. Nat. Rev. Immunol. 4:499-511.

31. Gordon, T.P., Kowanko, I.C., James, M., and Roberts-Thomson, P.J. 1985. Monosodium urate crystal-induced prostaglandin synthesis in the rat subcutaneous air pouch. Clin. Exp. Rheumatol. 3:291-296.

32. Matsukawa, A., et al. 1998. Analysis of the cytokine network among tumor necrosis factor alpha, interleukin-1beta, interleukin-8, and interleukin-1 receptor antagonist in monosodium urate crystalinduced rabbit arthritis. Lab. Invest. 78:559-569.

33. Getting, S.J., et al. 1997. Molecular determinants of monosodium urate crystal-induced murine perito- nitis: a role for endogenous mast cells and a distinct requirement for endothelial-derived selectins. J. Pharmacol. Exp. Ther. 283:123-130.

34. Liu-Bryan, R., Scott, P., Sydlaske, A., Rose, D.M., and Terkeltaub, R. 2005. Innate immunity conferred by Toll-like receptors 2 and 4 and myeloid differentiation factor 88 expression is pivotal to monosodium urate monohydrate crystal-induced inflammation. Arthritis Rheum. 52:2936-2946.

35. Hasan, U., et al. 2005. Human TLR10 is a functional receptor, expressed by B cells and plasmacytoid dendritic cells, which activates gene transcription through MyD88. J. Immunol. 174:2942-2950.

36. Heil, F., et al. 2004. Species-specific recognition of single-stranded RNA via toll-like receptor 7 and 8 . Science. 303:1526-1529.

37. Compton, T., et al. 2003. Human cytomegalovirus activates inflammatory cytokine responses via CD14 and Toll-like receptor 2. J. Virol. 77:4588-4596.

38. Liu-Bryan, R., Pritzker, K., Firestein, G.S., and Terkeltaub, R. 2005. TLR2 signaling in chondrocytes drives calcium pyrophosphate dihydrate and monosodium urate crystal-induced nitric oxide generation. J. Immunol. 174:5016-5023.

39. Yagnik, D.R., et al. 2004. Macrophage release of transforming growth factor beta 1 during resolution of monosodium urate monohydrate crystal-induced inflammation. Arthritis Rheum. 50:2273-2280.

40. Barabe, F., Gilbert, C., Liao, N., Bourgoin, S.G., and Naccache, P.H. 1998. Crystal-induced neutrophil activation VI. Involvement of FcgammaRIIIB (CD16) and CD11b in response to inflammatory microcrystals. FASEB J. 12:209-220.

41. Jaques, B.C., and Ginsberg, M.H. 1982. The role of cell surface proteins in platelet stimulation by monosodium urate crystals. Arthritis Rheum. 25:508-521.

42. Auwerx, J., Staels, B., Van Vaeck, F., and Ceuppens, J.L. 1992. Changes in IgG Fc receptor expression induced by phorbol 12-myristate 13 -acetate treatment of THP-1 monocytic leukemia cells. Leuk. Res. 16:317-327.

43. Martinon, F., Petrilli, V., Mayor, A., Tardivel, A., and Tschopp, J. 2006. Gout-associated uric acid crystals activate the NALP3 inflammasome. Nature. 440:237-241.

44. Chapman, P.T., et al. 1997. Endothelial activation in monosodium urate monohydrate crystalinduced inflammation: in vitro and in vivo studies on the roles of tumor necrosis factor alpha and interleukin-1. Arthritis Rheum. 40:955-965.

45. Armstrong, D.A., Major, J.A., Chudyk, A., and Hamilton, T.A. 2004. Neutrophil chemoattractant genes KC and MIP-2 are expressed in different cell populations at sites of surgical injury. J. Lenkoc. Biol. 75:641-648.

46. Terkeltaub, R., Baird, S., Sears, P., Santiago, R., and Boisvert, W. 1998. The murine homolog of the interleukin-8 receptor CXCR-2 is essential for the occurrence of neutrophilic inflammation in the air pouch model of acute urate crystal-induced gouty synovitis. Arthritis Rheum. 41:900-909.

47. Adachi, O., et al. 1998. Targeted disruption of the MyD88 gene results in loss of IL-1- and IL-18-mediated function. Immunity. 9:143-150.

48. Yamamoto, M., et al. 2002. Essential role for TIRAP in activation of the signalling cascade shared by 
TLR2 and TLR4. Nature. 420:324-329.

49. Yamamoto, M., et al. 2003. Role of adaptor TRIF in the MyD88-independent toll-like receptor signaling pathway. Science. 301:640-643.

50. Yamamoto, M., et al. 2003. TRAM is specifically involved in the Toll-like receptor 4-mediated MyD88-independent signaling pathway. Nat. Immunol. 4:1144-1150.

51. Takeuchi, O., et al. 2002. Cutting edge: role of Tolllike receptor 1 in mediating immune response to microbial lipoproteins. J. Immunol. 169:10-14.

52. Takeuchi, O., et al. 1999. Differential roles of TLR2 and TLR4 in recognition of gram-negative and gram-positive bacterial cell wall components. Immunity. 11:443-451.
53. Hoshino, K., et al. 1999. Cutting edge: Toll-like receptor 4 (TLR4)-deficient mice are hyporesponsive to lipopolysaccharide: evidence for TLR4 as the Lps gene product. J. Immunol. 162:3749-3752.

54. Takeuchi, O., et al. 2001. Discrimination of bacterial lipoproteins by Toll-like receptor 6. Int. Immunol. 13:933-940.

55. Hemmi, H., et al. 2002. Small anti-viral compounds activate immune cells via the TLR7 MyD88-dependent signaling pathway. Nat. Immunol. 3:196-200.

56. Hemmi, H., et al. 2000. A Toll-like receptor recognizes bacterial DNA. Nature. 408:740-745.

57. Zhang, D., et al. 2004. A toll-like receptor that prevents infection by uropathogenic bacteria. Science. 303:1522-1526.
58. Fitzgerald, K.A., et al. 2003. LPS-TLR4 signaling to IRF-3/7 and NF-kappaB involves the toll adapters TRAM and TRIF. J. Exp. Med. 198:1043-1055.

59. Latz, E., et al. 2004. TLR9 signals after translocating from the ER to CPG DNA in the lysosome. Nat. Immunol. 5:190-198.

60. Fitzmaurice, G.M., Laird, N.M., and Ware, J.H 2004. Applied longitudinal analysis. Wiley-Interscience. Hoboken, New Jersey, United States. 536 pp.

61. McLean, R.A., Sanders, W.L., and Stroup, W.W. 1991. A unified approach to mixed linear models. Am. Stat. 45:54-64.

62. Calvin, J.A. 1993. REML estimation in unbalanced multivariate variance components models using an EM algorithm. Biometrics. 49:691-701. 